\title{
Decitabine as a treatment choice for de novo acute basophilic leukemia: transient response-a case report
}

\author{
Lei Cao ${ }^{1,2,3}$, Rong Wang ${ }^{1,2,3}$, Yan Wang ${ }^{1,2,3}$, Si-Shu Zhao ${ }^{1,2,3}$, Hui Yang ${ }^{1,2,3}$, Ji Xu ${ }^{1,2,3}$, Qi-Qiang Long ${ }^{1,2,3,4}$, \\ Guang-Sheng $\mathrm{He}^{1,2,3}$, Jian-Yong $\mathrm{Li}^{1,2,3}$ \\ ${ }^{1}$ Department of Hematology, the First Affiliated Hospital of Nanjing Medical University, Jiangsu Province Hospital, Nanjing 210029 , China; ${ }^{2}$ Key \\ Laboratory of Hematology of Nanjing Medical University, Nanjing 210029, China; ${ }^{3}$ Collaborative Innovation Center for Cancer Personalized \\ Medicine, Nanjing 210029, China; ${ }^{4}$ Department of Hematology, Nanjing University of Chinese Medicine, The Second Hospital of Nanjing, Nanjing \\ 210000, China \\ Correspondence to: Dr. Guang-Sheng He, MD, PhD. Department of Hematology, the First Affiliated Hospital of Nanjing Medical University, Jiangsu \\ Province Hospital, Nanjing 210029, China; Key Laboratory of Hematology of Nanjing Medical University, Nanjing 210029, China; Collaborative \\ Innovation Center for Cancer Personalized Medicine, Nanjing 210029, China. Email: heguangsheng1972@sina.com.
}

\begin{abstract}
Acute basophilic leukemia (ABL), as a rare form of acute myeloid leukaemia (AML) accounts for $<1 \%$ of cases of AML. ABL has not been detected for encouragingly specific targets. Here we report a de novo fragile ABL case treated with decitabine based regimen with transient response even if overall survival was a 3-month. The case of a 79-year-old male who was complained of fever, rashes and cytopenia is reported in the current study. The diagnosis of ABL was identified due to characteristic cytomorphological features and immunophenotype of myeloid blast cells without the Philadelphia chromosome. The patient initially presented with short-term improvement with decitabine. Combination of decitabine and arsenic trioxide in second chemotherapy regimen didn't reverse the end of death with a 3 months overall survival. In conclusion, our study revealed that decitabine may be an efficient therapeutic option in ABL patients and warranted much more exploration in use.
\end{abstract}

Keywords: Acute basophilic leukemia (ABL); decitabine; therapy; a case report

Submitted Oct 23, 2019. Accepted for publication Dec 03, 2019.

doi: $10.21037 /$ tcr.2019.12.14

View this article at: http://dx.doi.org/10.21037/tcr.2019.12.14

\section{Introduction}

Acute basophilic leukemia (ABL), as a rare form of acute myeloid leukaemia (AML) in which basophils participate in primary differentiation, accounts for $<1 \%$ of cases of AML. The prognosis of ABL remains disappointing while most patients suffering from ABL died within 1 year. ABL has not been detected for encouragingly specific targets although arsenic trioxide, hydroxyurea or poly-chemotherapy was reported to be short-term efficient therapeutic option in de novo ABL (1). The 79-year-old male patient in our study was not candidate for intensive chemotherapy owing to his poor cardiopulmonary reserve capacity and Eastern Cooperative Oncology Group (ECOG) Performance status (PS) of 2 and Charlson scored 8. A 10-day schedule of decitabine was reported to be highly active and well tolerated, providing a possible framework upon which to build future combination therapy to improve outcomes for older unfit AML patients (2). In the present report, the specific case of an elderly male presenting with ABL, complained of fever and cytopenia, was treated with decitabine and then died of heart failure and infection with a total overall survival of 3 months. The report was confirmed by the Ethics Committee of Jiangsu Province Hospital and was performed on the basis of the Declaration of Helsinki. Written informed consent was attained from the patient's family. We present the following case in accordance with the CARE Guideline (3).

\section{Case presentation}

In May 2018, a 79-year-old male was admitted to the First 


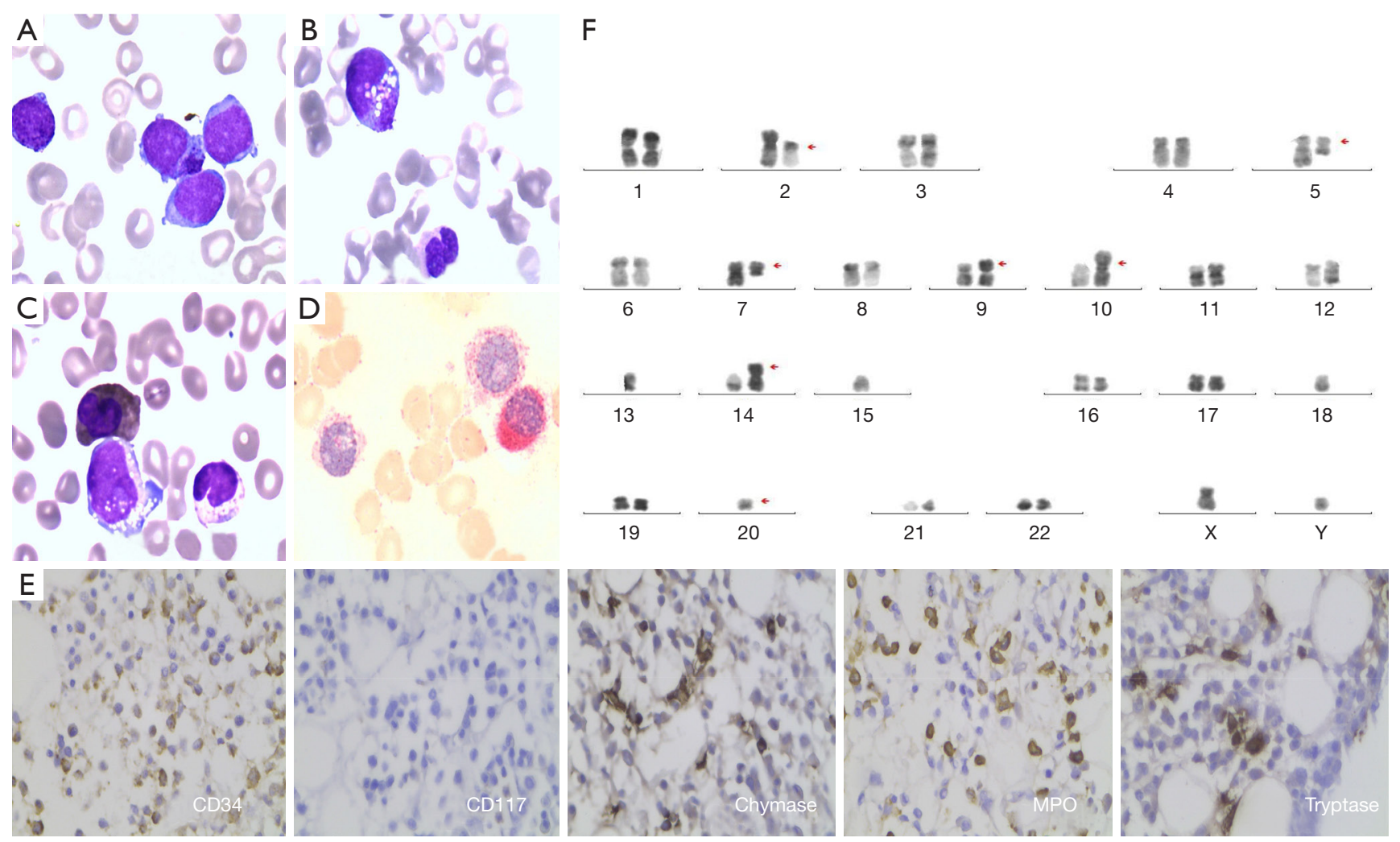

Figure 1 Illustrations. (A,B) Original magnification $\times 1,000$ : note the presence of immature basophils with purple-black granules gathering. (C) POX staining (original magnification $\times 1,000$ ): (negative). (D) PAS staining (original magnification $\times 1,000)$ : (positive). (E) Immunohistochemistry of the bone marrow biopsy confirmed CD34 +, MPO+, CD117-, Chymase ++ and Tryptase +++ (original magnification $\times 400)$ indicating ABL. (F) Chromosomal karyotype of the patient: 43,XY,der(2), del(5)(q15q35), del(7)(q21), der(10)t(10;15) $(\mathrm{q} 26 ; \mathrm{q} 25),-13,-15, \operatorname{del}(16)(\mathrm{p} 11),-20[9] / 42, \mathrm{XY}, \operatorname{del}(2)(\mathrm{p} 11), \operatorname{del}(5)(\mathrm{q} 15 \mathrm{q} 35), \operatorname{del}(7)(\mathrm{q} 21), \operatorname{der}(9 ; 15)(\mathrm{q} 10 ; \mathrm{q} 10), \operatorname{add}(10)(\mathrm{p} 11),-13, \operatorname{der}(14 ; 15)$ $(\mathrm{q} 10 ; \mathrm{q} 10),-15, \operatorname{del}(16)(\mathrm{p} 11),-18,-20, \operatorname{del}(20)(\mathrm{q} 13)[1]$.

Affiliated Hospital of Nanjing Medical University, Jiangsu Province Hospital, with repeated fever, cytopenia, weight loss (above $10 \%$ in 4 months), rashes and itches that had sustained for two weeks. He also showed past history of hypertension without medication treatment for 18 years and gastric cancer surgery without chemotherapy for more than 6 months. He has nothing special in family and psychosocial history without interventions. The initial physical examination showed notable pallor and mild lung dampness without hepatosplenomegaly and lymphadenopathy in May 2018.

Blood routine examination showed total white cell count of $1.71 \times 10^{9} / \mathrm{L}$, absolute neutrophils count of $0.67 \times 10^{9} / \mathrm{L}$, hemoglobin level of $60 \mathrm{~g} / \mathrm{L}$, platelet count of $47 \times 10^{9} / \mathrm{L}$, with $1 \%$ blast cells and $15 \%$ mature basophils. The antiENA antibody sets and anti-nuclear antibody typing sets revealed positive to anti-nuclear antibody titration
(ANA-T), anti-nuclear glycogen $\mathrm{P}$ protein antibody (A-RPP), anti-centromere B-protein antibody, and the antidouble-stranded DNA. On the set of the diagnosis of ABL, the sCD25 was tested at a higher titration with $20,456 \mathrm{pg} / \mathrm{L}$.

Bone marrow aspirate revealed $26 \%$ blasts including $10.4 \%$ basophilic granules showing that the abnormal cells were medium size, with a small amount of cytoplasm, pale blue, a round or elliptical nucleus, owing a fine sand shape on the nuclear chromosome and 2-4 nucleolus. Purple-black particles were found existing in some of the original cell cytoplasm (Figure 1). Besides, pathological hematopoietic like nuclear shrinkage, poor leaves, particle reduction, circular pole nucleus can be seen in the granule system. Flow cytometry showed $32 \%$ blast cells in bone marrow expressing CD34, CD117, CD13, CD33, CD38, CD81, CD58, CD123 and partial expressing CD11b, CD4 indicating 


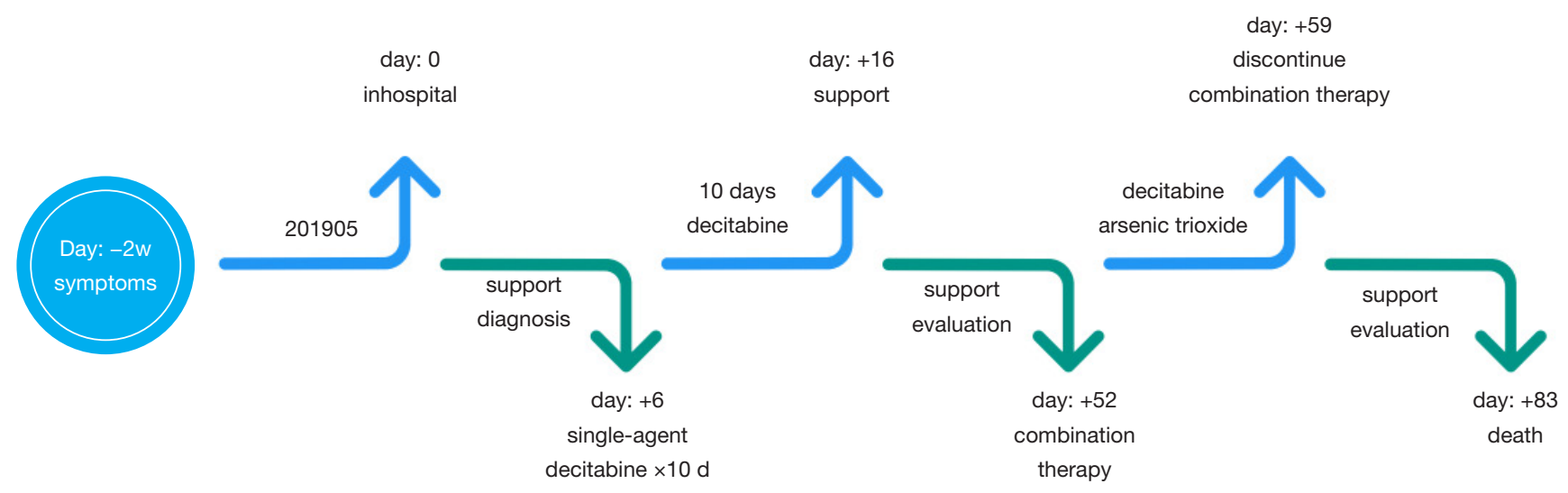

Figure $2 \mathrm{~A}$ timeline of the $\mathrm{ABL}$ case. $\mathrm{ABL}$, acute basophilic leukemia.

acute myeloid leukemia. The immunohistochemistry of the bone marrow biopsy confirmed CD34 +, MPO+, CD117-, Chymase ++ and Tryptase +++ indicating ABL (Figure 2). Besides, the cytogenetic examination verified $43, \mathrm{XY}, \operatorname{der}(2), \operatorname{del}(5)(\mathrm{q} 15 \mathrm{q} 35), \operatorname{del}(7)(\mathrm{q} 21), \operatorname{der}(10) \mathrm{t}(10 ; 15)$ (q26;q25),-13,-15, del(16)(p11),-20[9]/42,XY,del(2) (p11),del(5)(q15q35), del(7)(q21),der(9;15)(q10;q10),add(10) (p 11$),-13, \operatorname{der}(14 ; 15)(\mathrm{q} 10 ; \mathrm{q} 10),-15, \operatorname{del}(16)$ (p11),-18,-20, del(20)(q13)[1] predicting poor prognosis. The NGS of the patient proved mutation of DNMT3A (p.A128fs, $11.78 \%$ ) and ASXL1(p.G643fs, 5.95\%). Mutation of DNMT3A (p.A128fs) and ASXL1(p.G643fs) indicated frameshift somatic cell mutation in this case. In conclusion, we diagnosed him ABL, combined with cytopenia and pulmonary infection, and we didn't meet diagnostic challenges in his diagnosis. The above diagnostic tests including complex karyotype, ABL, comorbidity and age indicated poor prognosis.

Considering the status of $\mathrm{ABL}$, and the patient was too fragile with ECOG scored 2 and Charlson scored 8 to receive standard chemotherapy. He was treated with singleagent decitabine $20 \mathrm{mg} / \mathrm{m}^{2}$ ivd $\mathrm{qd} \times 10 \mathrm{~d}$ for the first cycle in timeline Figure 2. After 3 days of decitabine monotherapy, the vital signs of the patient including fever, rashes, itches and blood pressure were significantly relieved. The blood smear revealed basophilic cells decreased from 39\% to $15 \%$ while blast cells deceased from $2 \%$ to $1 \%$ after 5 days treatment.

During the process of demethylation therapy, rashes, pruritus and hypotension appeared in this patient and the symptoms were alleviated by loratadine and tranilast capsules. The skin lesions were disappeared gradually. The patient recovered from the combination of blood transfusion, G-CSF support, anti-infection therapy and intravenous nutrition during myelosuppression process although he experienced paroxysmal atrial fibrillation without hemodynamic change.

We conducted bone marrow puncture and biopsy and found $8.4 \%$ blast cells and $14.4 \%$ basophilic cells in day $10^{\text {th }}$ after the first cycle after informed consent of the patient.

The skin lesions appeared gradually from the day $36^{\text {th }}$ after the first cycle therapy. Considering the increased basophilic cells in bone marrow and peripheral blood, we thought that single decitabine didn't bring a longtime response and adjusted the therapeutic regimen. So the patient received the second therapy consisting of decitabine $\left(20 \mathrm{mg} / \mathrm{m}^{2} \mathrm{qd}\right)$ and arsenic trioxide $(0.15 \mathrm{mg}$ per $/ \mathrm{kg} / \mathrm{d})$, which had to discontinue at 7 th day since the severe pneumonia. After the second cycle of treatment, the blood smear revealed both of the blast and basophilic cells disappeared.

In the following half a month, the patient gradually appeared with irreversible heart failure, respiratory failure, renal insufficiency and somnolence and died in the end of July 2018, while his blood smear showed basophilic cells proportion was up to $18 \%$. The patient actively cooperated with the doctor to complete the above treatment even if he died in the end of July 2018.

\section{Discussion}

$\mathrm{ABL}$ is characterized by clinical manifestations of $\mathrm{AML}$ and 
abnormal proliferation of basophilic granules-containing immature cells. De novo acute basophilic leukemia, first reported by Wick et al. in 1982 (4), has been integrated as a distinct disease and defined as a kind of AML in which the primary differentiation is to basophilic cells (5). ABL is difficult to diagnose after excluding the other previously described groups including AML with recurrent genetic abnormalities, myelodysplasia-related changes, therapyrelated AML and chronic myeloid leukemia (CML). Peripheral smear should reveal basophilia and the bone marrow may demonstrate blast cells, often with immature basophilic granules and with or without mature basophils (6).

Common to other types of acute leukemia, patients suffering from $\mathrm{ABL}$ were complained of cytopenia due to bone marrow failure whether combining with circulating blasts or not. Besides, typical hyperhistaminaemia may lead to cutaneous involvement symptoms, organomegaly, lytic lesions, hypotension, et al. The clinical manifestations and cytogenetic pattern distinguish de novo ABL cases from those resulting from transformation of CML and from other AML subtypes with basophilia.

In a recent review (1), primary ABL is defined as follows: the proportion of myeloblasts and metachromatic blasts $20 \%$ and basophils (many basophils may be quite immature cells, presenting as metachromatic blasts, can been confirmed by immunophenotyping or electron microscopy) $40 \%$ of nucleated $\mathrm{BM}$ or $\mathrm{PB}$ cells without preceding or underlying BM neoplasm. Besides, all suspected basophilic leukemia cases should accept conventional karyotyping, FISH, sequencing of typically mutated genes [myelodysplastic syndrome (MDS)/AML panel, MPN panel], probing for detection of $\mathrm{Ph}$, fusion gene of BCRABL1 and FIP1L1-PDGFR1 and JAK2 V617F by PCR.

Due to its sporadic cases, no consistent chromosomal abnormality has been confirmed even if a recurrent $\mathrm{t}(\mathrm{X} ; 6)(\mathrm{p} 11.2 ; \mathrm{q} 23.3)$ resulting in MYBGATA1 $(7,8), \mathrm{t}(3 ; 6)$ ( $21 ; \mathrm{p} 21)$, abnormalities involving $12 \mathrm{p}$ and so on in several cases. But AML with $\mathrm{t}(6 ; 9)(\mathrm{p} 23 ; \mathrm{q} 34.1)$ is usually excluded owing to its association with BCR-ABL1. And it was reported add (3)(q12) as a novel cytogenetic aberration in ABL by Koya et al. in 2017 (9).

According to the clinical manifestation and the results of flow cytometry, morphology and genetics, the patient was diagnosed of ABL definitely. It is interesting that the cytogenetic examination verified 43,XY,der(2), del(5)(q15q35), del(7)(q21),der(10)t(10;15) (q26;q25),-13,-15, del(16)(p11),-20[9]/42,XY,del(2) (p11), del(5)(q15q35), del(7)(q21),der(9;15)(q10;q10), add(10)
( p 11$),-13$, der $(14 ; 15)($ q $10 ;$ q 10$),-15, \operatorname{del}(16)$ (p11),-18,-20,del(20)(q13)[1] which is different to the existing reports including abnormality of chromosome 3, 6 and 12. And among them, del5, del7 and -20 are common karyotype abnormalities of MDS. The bone marrow smear of the elderly patient shows morphologically significant pathological hematopoiesis, indicating that the patient was more likely developed from MDS. Besides, the next generation sequence of DNMT3A (p.A128fs, 11.78\%) and ASXL1 (p.G643fs, 5.95\%) existing much more in MDS and AML further support the speculation.

There is little research on survival with this rare type of acute leukaemia. The cases suffered from ABL reported have generally been associated with a poor prognosis and been regarded as candidates for allo-stem cell transplantation.

Our patient was not eligible for high-dose therapy and treated with decitabine combined with best response at partial remission.

According to the result of clinical trial NCT00260832, decitabine improved overall survival in older adults with newly diagnosed AML (10). Besides, a phase II clinical trial with single-agent decitabine targeting elderly untreated AML patients showed $47 \%$ complete remission with normal karyotype and 50\% in complex karyotype (11). And the research pointed out that expression of DNMT3a should be validated as a stratification tool in selection of older AML patients for decitabine-based treatments (11). NCT00538876, an open-label phase 1 study, concluded that epigenetic priming with decitabine prior to standard induction chemotherapy can be safely delivered in an attempt to improve response rates (12). Considering of the fragile condition of the patient, we designed the first cycle of mono-drug decitabine at a low dosage and observed the early response in peripheral and bone marrow.

In two phase 2 clinical studies, major responses were observed in MDS including higher-risk group with singleagent $\mathrm{As}_{2} \mathrm{O}_{3}(13,14)$. Furthermore, $\mathrm{As}_{2} \mathrm{O}_{3}$ shows efficacy in AML patients in much more researches. The combination of decitabine and arsenic trioxide was proved to significantly increasing the response rate in MDS patients in a phase I study (15), while the synergistic effect of inhibition of methylation and induction of malignant leukemia stem cell apoptosis might make the contribution (16).

Considering the synergism of decitabine and $\mathrm{As}_{2} \mathrm{O}_{3}$, we adopted the regimen of decitabine and $\mathrm{As}_{2} \mathrm{O}_{3}$ in the second cycle for the salvage treatment. The blood smear and blood routine test revealed the quick response and remission of 
symptoms even if the duration was transient.

In conclusion, our study revealed that decitabine may be an efficient chemotherapeutic option in ABL patients even of short response duration without remission and warranted much more exploration in this drug. These ABL patients may be candidates for some experimental drugs or palliative cytoreductive treatment and new combination chemotherapy. We present the following case in accordance with the CARE Guideline.

\section{Patient perspective}

During the initial therapy of mono-drug decitabine and other supportive treatment, the patient felt better while his rashes, pruritus and hypotension were alleviated and improved physical strength and appetite. Once his malignant ABL cells increased, he felt some above symptoms coming again and showed full confidence to the combination of decitabine and $\mathrm{As}_{2} \mathrm{O}_{3}$ even if his overall survival was a 3 -month.

\section{Acknowledgments}

We thank all members of our group for their efforts and enthusiasm.

Funding: None.

\section{Footnote}

Conflicts of Interest: All authors have completed the ICMJE uniform disclosure form (available at http://dx.doi. org/10.21037/tcr.2019.12.14). The authors have no conflicts of interest to declare.

Ethical Statement: The authors are accountable for all aspects of the work in ensuring that questions related to the accuracy or integrity of any part of the work are appropriately investigated and resolved. All procedures performed in studies involving human participants were in accordance with the Declaration of Helsinki (as revised in 2013). Informed consent for the publication of the report, and the accompanying images, was provided by the patient and his son. The submission version of the report was read by his son, and the report's content was confirmed as being correct to the best of his knowledge.

Open Access Statement: This is an Open Access article distributed in accordance with the Creative Commons
Attribution-NonCommercial-NoDerivs 4.0 International License (CC BY-NC-ND 4.0), which permits the noncommercial replication and distribution of the article with the strict proviso that no changes or edits are made and the original work is properly cited (including links to both the formal publication through the relevant DOI and the license). See: https://creativecommons.org/licenses/by-nc-nd/4.0/.

\section{References}

1. Valent P, Sotlar K, Blatt K, et al. Proposed diagnostic criteria and classification of basophilic leukemias and related disorders. Leukemia 2017;31:788-97.

2. Blum W, Garzon R, Klisovic RB, et al. Clinical response and miR-29b predictive significance in older AML patients treated with a 10-day schedule of decitabine.Proc Natl Acad Sci U S A 2010;107:7473-8.

3. Riley DS, Barber MS, Kienle GS, et al. CARE 2013 Explanations and Elaborations: Reporting Guidelines for Case Reports. J Clin Epidemiol 2017;89:218-35.

4. Wick MR, Li CY, Pierre RV. Acute nonlymphocytic leukemia with basophilic differentiation. Blood 1982;60:38-45.

5. Vardiman JW, Thiele J, Arber DA, et al. The 2008 revision of the World Health Organization (WHO) classification of myeloid neoplasms and acute leukemia: rationale and important changes. Blood 2009;114:937-51.

6. Peterson LC, Parkin JL, Arthur, DC, et al. Acute basophilic leukemia: A clinical, morphologic and cytogenetic study of eight cases. Am J Clin Pathol 1991;96:160-70.

7. Dastugue N, Duchayne E, Kuhlein E, et al Acute basophilic leukaemia and translocation $\mathrm{t}(\mathrm{X} ; 6)(\mathrm{p} 11 ; \mathrm{q} 23)$. Br J Haematol 1997;98:170-6.

8. Quelen C, Lippert E, Struski S, et al. Identification of a transforming MYB-GATA1 fusion gene in acute basophilic leukemia: a new entity in male infants. Blood 2011;117:5719-22.

9. Koya J, Ibaraki T, Yamazaki I, et al. Acute basophilic leukemia with $\operatorname{add}(3)(\mathrm{q} 12)$ accompanied by histamine excess symptoms. Ann Hematol 2017;96:1197-9.

10. Mayer J, Arthur C, Delaunay J, et al. Multivariate and subgroup analyses of a randomized, multinational, phase 3 trial of decitabine vs treatment choice of supportive care or cytarabine in older patients with newly diagnosed acute myeloid leukemia and poor- or intermediate-risk cytogenetics. BMC Cancer 2014;14:69.

11. Blum W, Garzon R, Klisovic RB, et al. Clinical response 
and miR-29b predictive significance in older AML patients treated with a 10-day schedule of decitabine. Proc Natl Acad Sci U S A 2010;107:7473-8.

12. Scandura JM, Roboz GJ, Moh M, et al. Phase 1 study of epigenetic priming with decitabine prior to standard induction chemotherapy for patients with AML. Blood 2011;118:1472-80.

13. Schiller GJ, Slack J, Hainsworth JD, et al. Phase II multicenter study of arsenic trioxide in patients with myelodysplastic syndromes. J Clin Oncol 2006;24:2456-64.

Cite this article as: Cao L, Wang R, Wang Y, Zhao SS, Yang H, $\mathrm{Xu}$ J, Long QQ, He GS, Li JY. Decitabine as a treatment choice for de novo acute basophilic leukemia: transient response-a case report. Transl Cancer Res 2020;9(2):1288-1293. doi: 10.21037/ tcr.2019.12.14
14. Vey N, Bosly A, Guerci A, et al. Arsenic trioxide in patients with myelodysplastic syndromes: a phase II multicenter study. J Clin Oncol 2006;24:2465-71.

15. Welch JS, Klco JM, Gao F, et al. Combination decitabine, arsenic trioxide, and ascorbic acid for the treatment of myelodysplastic syndrome and acute myeloid leukemia: a phase I study. Am J Hematol 2011;86:796-800.

16. Chen G, Wang Y, Huang H, et al. Combination of DNA methylation inhibitor 5-azacytidine and arsenic trioxide has synergistic activity in myeloma. Eur J Haematol 2009;82:176-83. 